\title{
Motivo de abandono del tratamiento desde la perspectiva del paciente con un trastorno de la conducta alimentaria
}

\section{Reason for treatment abandonment from the perspective of the patient with an eating disorder}

Recepción: 17 Septiembre 2015 | Aprobación: 17 Septiembre 2016

\author{
María Y. Vellisca** \\ Universidad de Zaragoza, España \\ José I. Latorre*** \\ Hospital Obispo Polanco, España \\ Santos Orejudo ${ }^{* * * *}$ \\ Universidad de Zaragoza, España \\ Andrés Gómez del Barrio****** \\ Hospital Universitario "Marqués de Valdecilla", \\ España \\ Laura Moreno \\ Great Ormond Street Hospital for children NHS \\ Foundation Trust, United Kingdom \\ Irene Checa Esquiva******* \\ Universidad de Valencia, España
}

***Departamento de Psicología y Sociología. Doctora en psicología. Correspondencia: C/ Ciudad Escolar, s/n, 44003, Teruel. Correo electrónico: neuropsicol@gmail.com

***Unidad de Salud Mental. Doctor en psicología. Correspondencia: Avenida Ruiz Jarabo s/n 44001 Teruel.

*****Departamento de Psicología y Sociología. Doctor en psicología. Correspondencia: Av San Juan Bosco, 7, 50009.

*****Unidad de Trastornos de la conducta Alimentaria, Servicio de Psiquiatría. Doctor en medicina. Correspondencia: Avda. Valdecilla s/n 39002, (Santander).

******Departament of Child and Adolescent Mental Health. Licenciada en psicología. Correspondencia: Great Ormond St, London WC1N 3JH, UK.

********Departamento y Metodología y Ciencias del Comportamiento. Facultad de Psicología. Doctora en psicología Correspondencia: Av. Blasco Ibáñez, 21, 46010-Valencia.

\section{RESUMEN}

Los tratamientos psicológicos y farmacológicos para los trastornos de la conducta alimentaria comportan costes personales y sanitarios. En este trabajo estudiamos los diferentes motivos que pueden estar asociados al abandono en ambos tipos de tratamientos en pacientes diagnosticados con un trastorno de la conducta alimentaria, así como el tipo de abandono: abandono tras evaluar, abandono precoz y tardío. Por otro lado, analizamos si existe alguna relación entre el diagnóstico y el tipo de abandono. Se examinaron diversas características (edad, sexo, diagnóstico, motivos y tipos de abandono, y satisfacción respecto a la unidad de trastornos de la conducta alimentaria) en 212 pacientes atendidos en un servicio especializado en el tratamiento de trastornos de la conducta alimentaria durante un periodo de dos años. Nuestros datos apoyan la hipótesis de que el tipo de abandono de los tratamientos más frecuente es de tipo tardío, motivado por la mejoría del trastorno de la conducta alimentaria, mientras que no se verifica que exista relación alguna entre el diagnóstico y el tipo de abandono. Nuestros resultados indican que los motivos de abandono son heterogéneos. Se pueden establecer dos perfiles diferenciados de abandono; uno ligado a la mejoría y a los impedimentos físicos temporales, y otro relacionado con motivos diversos.

Palabras clave

trastornos de la conducta alimentaria, abandono del tratamiento, motivos de abandono, tipos de abandono.

ABSTRACT 
Psychological and pharmacological treatments for eating disorder involve personal and health costs. In this paper we study the various reasons that may be associated to abandonment in both types of treatments in patients diagnosed with a disorder of eating behavior and the kind of neglect: neglect after evaluation, early and late abandonment. On the other hand, we analyze whether there is any relationship between the diagnosis and the type of abandonment. Various characteristics (age, sex, diagnosis, motives and types of neglect, and satisfaction with the unit eating disorder) in 212 patients treated at a specialized service in the treatment of eating disorder were examined during a period of two years. Our data support the hypothesis that the rate of abandonment of the most common treatments is delayed type, motivated by the improvement of the disorder of eating behavior, while no verified that there is some relationship between the diagnosis and the type of abandonment. Our results indicate that the reasons for dropping out are heterogeneous. You can set two different profiles of dropouts, one linked to improvement and to temporary disability, and other related to various reasons.

Keywords

eating disorders, treatment discontinuation, dropout reasons, dropout types.

Para citar este artículo: Vellisca, M., Latorre, J.I., Orejdo, S., Gómez del Barrio, A., Moreno, L., \& Checa Esquiva, I. (2016). Motivo de abandono del tratamiento desde la perspectiva del paciente con un trastorno de la conducta alimentaria. Universitas Psychologica, 15 (4). http://dx.doi. org/10.11144/Javeriana.upsy-15-4.matp

\section{Introducción}

Los trastornos alimentarios se caracterizan por alteraciones graves de la conducta alimentaria. El Manual Diagnóstico y Estadístico de Trastornos Mentales (DSM-IV-TR, American Psychiatric Association, 2002) clasifica los trastornos de la conducta alimentaria (TCA) en tres categorías: anorexia nerviosa (AN), bulimia nerviosa (BN) y trastorno de la conducta alimentaria no especificado (TCANE). Las dos primeras categorías son trastornos específicos, y la tercera categoría incluye los casos que no cumplen todos los criterios diagnósticos de $\mathrm{AN}$ o $\mathrm{BN}$, pero en los que sí existe una alteración de la conducta alimentaria. La AN se caracteriza por una pérdida progresiva de peso, ocasionada por la propia persona, a través de la disminución o evitación de la ingesta. La característica psicopatológica fundamental de este trastorno, al igual que ocurre con el resto de los TCA, es la distorsión de su imagen corporal, provocando una sobreestimación de su figura o peso corporal (Vellisca, Orejudo, \& Latorre, 2012). La BN se identifica por una preocupación continua por la comida, con deseos irresistibles de comer, presentando episodios recurrentes de ingesta de grandes cantidades de alimentos, en un espacio corto de tiempo y con un sentimiento de pérdida de control, de no poder parar de comer. Además, estos episodios suelen estar seguidos por conductas compensatorias inapropiadas como el ayuno, el vómito provocado, el abuso de laxantes, diuréticos, anorexígenos o el ejercicio excesivo (Gómez del Barrio, Gaite, Carral, Herrero, \& Vázquez-Barquero, 2012). La tercera categoría se describe por manifestar alteraciones de la ingesta que no cumplen todos los criterios necesarios para poder diagnosticar un trastorno específico. Suelen ser pacientes con sintomatología propia de la AN o BN pero que no cumplen con todos los criterios para ser un cuadro completo.

Las cifras de prevalencia actualmente de los TCA y en particular de la AN se estima alrededor del $0.9 \%$ en mujeres y del 0.3 en hombres; la de la BN sería aproximadamente de $1.5 \%$ en población femenina y $0.5 \%$ en población masculina; y el trastorno por atracón afectaría a cerca del $3.5 \%$ de las mujeres frente al $2 \%$ de los hombres (Hoek, 2006; Hoek \& Van Hoeken, 2003; Hudson, Hiripi, Pope, \& Kessler, 2007) La incidencia de casos de AN han pasado de ser 0.37 y 0.64 pacientes por año por cada 100.000 habitantes entre los años 60 y los años 70 (Jones, Fox, Babigan, \& Hutton, 1980; Kendell, Hall, Halley, \& Babigan, 1973), a situarse en torno al 8 y 8.6 (Currin, Schmidt, Treasure, \& Jick, 2005; Hoek, 2006; Hoek \& Van Hoeken, 2003).

A lo largo de estos últimos años se han llevado a cabo diferentes estudios (López \& Treasure 2011; Ryan \& Deci, 2000; Vandereychken, Soenens, \& Vansteenkiste, 2009; Waller et al., 2009) con el objetivo de conocer cuáles son las 
causas que llevan al paciente a que abandone el tratamiento, sin embargo, no se ha profundizado en cuáles son los motivos que conducen al abandono del tratamiento desde el punto de vista subjetivo del propio paciente con TCA.

Una gran parte de la población que sufre de estos trastornos no consultan directamente sobre ello ya que en general, la conciencia de enfermedad y la motivación para el cambio son escasas a pesar de que la salud física y psicológica se encuentre severamente amenazada por la enfermedad (López \& Treasure, 2011). Como consecuencia, los pacientes que presentan enfermedades crónicas y que no están en tratamiento, tienden a utilizar más recursos de otros servicios, tales como la atención médica, la atención hospitalaria, las emergencias, la fisioterapia, etc., frente a los pacientes que si están en tratamiento (Watson, Fursland, \& Byrne, 2013). En cuanto a aquellos sujetos que sí están en tratamiento pero que, sin embargo, abandonan, llegan a presentar un peor pronóstico del trastorno a largo plazo (Beumont, Russel, \& Touyz, 1993; Pike, 1998). Por otro lado, no hay que olvidar que la no adherencia al tratamiento suele ser un motivo de abandono del mismo, siendo este un problema real y poco estudiado. López y Treasure (2011) informan que uno de los motivos del abandono al tratamiento en pacientes con $\mathrm{AN}$ y BN podría ser la escasa efectividad del tratamiento, y a diferencia de otros trastornos de salud mental, la motivación para recuperarse es muy baja en pacientes con TCA. Por ello, la motivación es el principal motor para la recuperación de cualquier trastorno y se ha demostrado que las terapias convencionales fallan cuando la motivación es baja. Sin embargo, los motivos de abandono del tratamiento son diversos, entre ellos está la responsabilidad que recae en el paciente $y$ en sus propias elecciones. Ryan y Deci (2000) refieren que la voluntad y la autodeterminación son factores importantes que influyen en la motivación, la presión externa y las decisiones tomadas por otras personas, no la favorecen y en ocasiones esto desemboca en el abandono del tratamiento. Sin embargo, la eficacia del tratamiento es otra variable a considerar, si se conjuga este con la percepción de autonomía y la voluntad del paciente, llega a favorecer la continuidad y el cambio terapéutico (Vandereychken et al., 2009). Cuando los pacientes son totalmente responsables en la toma de decisiones, en cuanto al inicio y permanencia en el tratamiento, es menos probable que abandonen el mismo y por consiguiente, la probabilidad de que realicen progresos terapéuticos será mayor en comparación con cuando se sienten obligados a hacerlo (Zuroff, Koestner, \& Moskowitz, 2007). Waller et al. (2009) manifiestan que los abandonos precoces pueden estar relacionados con la impulsividad, mientras que los abandonos tardíos estarían relacionados con el progreso en el tratamiento y por las dificultades del mismo, como por ejemplo, la ganancia de peso.

El primer objetivo del presente trabajo es identificar los distintos tipos de abandono que se da en pacientes con TCA, basándonos en la clasificación realizada por los autores Waller et al. (2009). El abandono tras evaluar, incluye a los pacientes que han asistido al menos una vez al servicio y han sido evaluados, pero por su propia iniciativa y contrariando el consejo profesional, han salido del tratamiento antes de su comienzo. El abandono precoz comprende aquellos pacientes que han acudido al menos a tres consultas y el abandono tardío acoge al resto de pacientes que han interrumpido de forma voluntaria el tratamiento. El segundo objetivo es conocer cuáles son los motivos que pueden desencadenar el abandono del tratamiento desde la perspectiva subjetiva en pacientes diagnosticados con un TCA.

Para cumplir con los objetivos, se seleccionaron todos los pacientes diagnosticados con un TCA y que cumplían con todos los criterios del DSM-IV-TR (APA, 2002) para la AN, la BN y el TCANE. Se recogieron variables antropométricas (edad y sexo), diagnóstico, motivos y tipos de abandono, así como los aspectos de satisfacción respecto a la UTCA (Unidad de los Trastornos de la Conducta Alimentaria). Los resultados encontrados apoyan las hipótesis en cuanto a que los abandonos más frecuentes, son los de tipo 
María Y. Vellisca, José I. Latorre, Santos Orejudo, Andrés Gómez del Barrio, Et al.

tardío, motivados por la mejoría del trastorno. No se halló relación entre el diagnóstico y el tipo de abandono.

\section{Método}

\section{Participantes}

Del total de los 212 pacientes recibidos en la UTCA durante un periodo de dos años y que reunían las características señaladas, 87 fueron catalogados como abandonos. De dicha muestra, 23 no fueron localizados, 5 habían sido dados de alta y 5 seguían en tratamiento.

Por lo tanto, la muestra final se compuso de 54 sujetos diagnosticados con un TCA, de los cuales 52 eran mujeres y 2 varones. Sobre dicho total, 38 (70.4\%) fueron diagnosticados de BN, de los cuales 7 padecían una $\mathrm{BN}$ no purgativa $\mathrm{y}$ uno, BN purgativa. La muestra restante, estuvo conformada por 14 sujetos $(25.9 \%)$ que presentaban AN, de los cuales ocho eran casos de TCANE; cuatro, de AN restrictiva; y dos, de AN purgativa. Los dos casos restantes eran comedores nocturnos.

El rango de edades estuvo comprendido entre los 16 y 60 años, cuya $M=29.10$ ( $D T: 11.09$ ) con un IMC de $M=27.90$ ( $D T: 9.5$ ) y una edad media de inicio del TCA de 22.55 ( DT: 9.7).

\section{Variables $e$ instrumentos}

En la recolección de los datos se tuvo en cuenta el sexo, la edad, el diagnóstico, los motivos de abandono y algunos aspectos de satisfacción respecto a la UTCA. Para clasificar los tipos de abandono, se cuantificó las veces que los pacientes acudieron a consulta, y si iniciaron o no el tratamiento prescrito por los facultativos (psicólogo y psiquiatra) de la UTCA. Aquellos pacientes que al menos acudieron una vez al servicio y que fueron evaluados sin iniciar el tratamiento fueron clasificados como abandono tras evaluar ; la categoría abandono precoz comprendió aquellos pacientes que acudieron al menos a tres consultas e iniciaron el tratamiento; mientras que la de abandono tardío recogió al resto de pacientes que asistieron a la unidad en más de tres ocasiones y que mantuvieron el tratamiento.

\section{Procedimiento}

Este estudio se enmarca dentro de otro estudio longitudinal más amplio, dirigido a la detección y tratamiento precoz de pacientes con TCA con un primer episodio de la enfermedad y nunca tratados anteriormente mediante el programa de Detección Temprana en Cantabria de Trastornos Alimentarios (Gómez del Barrio et al., 2012). Los participantes fueron derivados a la UTCA del servicio de psiquiatría en los dos últimos años. Se seleccionaron a todos los sujetos que cumplían los criterios diagnósticos con TCA según el DSM-IV- TR (APA, 2002); AN restrictiva y AN compulsiva purgativa, BN purgativa y BN no purgativa y TCANE. Todos fueron informados de los objetivos del presente estudio concediendo su consentimiento para tal fin.

La información recopilada se obtuvo a través de la historia clínica y mediante una entrevista semiestructurada que fue elaborada por el coordinador de la UTCA para esta investigación. La entrevista se llevó a cabo por una psicóloga de dicha unidad de manera telefónica, con una duración media de diez minutos. Mediante esta última, se recopilaron los posibles motivos de abandono del tratamiento, mediante preguntas con respuestas alternativas que incluían características del tratamiento, la relación terapéutica, los impedimentos físicos-temporales, los impedimentos personales (embarazos o enfermedades), la falta de mejoría, la dejadez, la pérdida de cita, los sentimientos de vergüenza y la desmotivación.

\section{Análisis estadísticos}

Para analizar las variables recogidas en esta investigación se han usado los procedimientos habituales de análisis de variables categóricas, los análisis de frecuencias, las tablas de contingencias y por último, se han aplicado 
técnicas de análisis de clusters para crear grupos de participantes según el perfil que presentaban. El método seguido en este caso ha sido el bietápico. Los cálculos se realizaron mediante el programa Statistical Package for the Social Sciences (SPSS, 2010) versión 19.0.

\section{Resultados}

En el análisis de datos se utilizó el estadístico descriptivo de frecuencias. En la Tabla 1 puede verse el porcentaje de los motivos de abandono. Estos se distribuyen de la siguiente manera: abandonos por mejoría, el $18.5 \%$; por características del tratamiento y de la relación terapéutica, $7.4 \%$; por impedimentos físicotemporales, $20.4 \%$. El resto de los motivos fueron diversos (impedimentos personales, embarazos, enfermedades, falta de mejoría, dejadez, pérdida de cita, sentimientos de vergüenza o desmotivación), sumando entre todos el 53.7\%. A su vez, se analizó la frecuencia de los diferentes tipos de abandono con porcentajes de un $7.4 \%$ en los abandonos tras la evaluación, del $20.4 \%$ en los abandonos precoces y del $72.2 \%$ en los abandonos de carácter tardío (Tabla 2).

TABLA 1

Motivos de abandono

\begin{tabular}{lll}
\hline & Frecuencia & Porcentaje \\
\hline Mejoría & 10 & 18.5 \\
Características del tratamiento & 4 & 7.4 \\
Impedimentos físico-temporales & 11 & 20.4 \\
& & \\
Otros & 29 & 53.7 \\
Total & 54 & 100 \\
\hline
\end{tabular}

Fuente: elaboración propia
TABLA 2

Tipos de abandono

\begin{tabular}{lcc}
\hline & Frecuencia & Porcentaje \\
\hline Abandono tras evaluar & 4 & 7.4 \\
Abandono precoz & 11 & 20.4 \\
Abandono tardío & 39 & 72.2 \\
Total & 54 & 100 \\
\hline
\end{tabular}

Fuente: elaboración propia

La mayor parte de las personas que han abandonado son casos de $\mathrm{BN}, 38$ (70.4\%) De estos, los más frecuentes son el grupo de no identificados $(55.6 \%)$ del total, seguidos a distancia por los casos de $\mathrm{BN}$ no purgativa 7 casos $(13 \%)$ y purgativa 1 caso $(1.9 \%)$. Catorces casos $(25.9 \%)$ se corresponden con diagnósticos de AN, siendo la no especificada el grupo más numeroso de 8 casos (14.8\%), seguido de la restrictiva 4 casos $(7.4 \%)$ y de la purgativa 2 casos $(3.7 \%)$. Los dos casos restantes para llegar a los 54 , son comedores nocturnos.

En relación con el momento del abandono, la mayor parte lo hace a lo largo de la intervención $(72.2 \%, \mathrm{n}=39)$, un $20.4 \%(\mathrm{n}=11)$ ha acudido al menos a tres consultas y únicamente 4 casos, $(74.4 \%)$ lo han hecho antes de iniciar el tratamiento, es decir, al acabar el proceso de evaluación. No hay relación entre el diagnóstico y el tipo de abandono $\left(\#^{2}=7.464, p=0.825\right)$ En el momento de volver a contactar, $53.7 \%$ indican encontrarse bien, un $18.5 \%$ mejor, un $7.4 \%$ igual y un 14.8 mal o bastante mal. Tres casos deciden no contestar. No hay relación entre el momento en el que se produce el abandono y el estado actual $\left(\#^{2}=1.467, p=0.993\right)$. No hay relación entre el estado en el que se encuentran las pacientes y el tipo de diagnóstico o el momento en el que se produce el abandono.

En cuanto a los motivos que se han señalado como justificación del abandono, en una primera codificación aparece la mejoría en el $18.5 \%$, 
María Y. Vellisca, José I. Latorre, Santos Orejudo, Andrés Gómez del Barrio, Et al.

las características del tratamiento y la relación terapéutica $7.4 \%$ y los impedimentos físicostemporales $20.4 \%$, otros motivos, fueron de un $53.7 \%$. Estos últimos recogían motivos tales como impedimentos personales, embarazos o enfermedades $(11 \%)$, la no mejoría (9\%), la dejadez $(11.1 \%)$, la pérdida de cita $(9.3 \%)$, los sentimientos de vergüenza y la desmotivación.

No hemos encontrado relaciones entre las variables anteriores y los motivos alegados, siendo la más consistente la que establece la asociación entre el motivo del abandono y el estado en el que se encuentra la paciente en el momento del contacto $\left(\#^{2}=26.38, p=0.009\right.$, $\#=0.699$ ). Así, entre los que ahora dicen estar mejor aparecen motivos para el abandono como la mejoría y son menos frecuentes los "otros" motivos. Por su parte, los que están igual suelen haber alegado otros motivos para abandonar y los que son categorizados como "no sabe, no contesta" suelen haber alegado características del tratamiento y del entorno terapéutico.

Mediante el análisis cluster hemos intentado determinar posibles tipologías de perfiles de abandono. Así, el programa estadístico nos ofrece una solución con dos clusters que agrupan a 50 de los 54 casos seleccionados, 25 en cada uno de los grupos. Las variables que permiten discriminar entre ambos grupos son el motivo de abandono, el diagnóstico y haber respondido afirmativamente al motivo de abandono relacionado con la mejoría, con la no mejoría y con los impedimentos físicos temporales. Así, el cluster 1 queda definido por personas que mayoritariamente alegan abandonar por mejoría o por problemas físicos temporales, el $100 \%$ de los casos de este grupo están en este cluster. Este primer cluster suele tener a personas con AN, el $85.7 \%$ de los casos de este trastorno se ubican en este cluster, con perfiles específicos de AN purgativa, el $71.4 \%$ de los casos, que alegan abandonar por mejoría o por impedimentos físicos temporales, $\mathrm{y}$ en ningún caso por falta de mejoría.

El segundo cluster, con otras 25 personas, presenta como característica principal, abandonos por motivos relacionados con el tratamiento y el terapeuta, todos los casos en los que se codificó esta respuesta están en este cluster, o por otros motivos peor identificados el $77.8 \%$ de los casos de este motivo presentan igualmente un perfil diferencial en el diagnóstico, ya que la mayor parte son casos de $\mathrm{BN}$, en concreto, 21 casos presentan el diagnóstico de $\mathrm{BN}$ no especificada. Igualmente, en ningún caso se alegó la mejoría como motivo de abandono o los impedimentos físicos temporales, y todos los casos en los que se alegaron motivos de falta de mejoría están en este segundo cluster.

Por último, aun habiendo contactado con pacientes que han abandonado, la mayor parte de ellos refirieron que volverían a pedir ayuda en el caso de necesitarla $(96.2 \%)$ y recomendarían la unidad de tratamiento (94.4\%), mientras que el resto $\mathrm{n}=3$ no reseñaron nada al respecto.

\section{Discusión}

Los resultados finales y acordes a nuestra hipótesis indican que los pacientes abandonaban el tratamiento principalmente por mejoría. El resto de datos revelan que las características del tratamiento, la relación con el terapeuta o el no cumplimiento de las expectativas generadas por parte de los usuarios o por otras causas, comorbilidad y/o la desesperanza, son motivos de abandono. Por otro lado, también se presentaron otros motivos; los embarazos, las enfermedades propias o de otros familiares, la vergüenza, los impedimentos físico-temporales, la distancia y la falta de tiempo, todos ellos también los refieren los pacientes como razones para abandonar los tratamientos. Nuestros datos evidencian que un $72 \%$ de los abandonos son tardíos, seguidos por un $20.4 \%$ de abandonos de carácter precoz y un $7.4 \%$ de abandono tras evaluar.

La mayor parte de los estudios manifiestan que el abandono de la terapia antes de completarla, es un predictor de mal pronóstico de mejoría del trastorno (Beumont et al., 1993; Pike, 1998; Waller et al., 2009), sin embargo, en nuestra muestra el $18.5 \%$ indicaba que habían dejado de acudir a la consulta porque se encontraba mejor, 
mostrándose en este caso un factor de buen pronóstico. Estos resultados coinciden con los hallados por Velázquez, Sosa, Lara, \& Sentíes (2013), estudio en el que investigaron en una muestra clínica de 314 pacientes pertenecientes a una institución de salud mental, los diferentes tipos de abandono al tratamiento, encontrando que un alto porcentaje de sujetos, abandonaban el mismo por sentirse mejor $(19.6 \%)$.

Un $25.9 \%$ de los pacientes participantes, señalaron que los motivos de abandono de los tratamientos fueron los impedimentos físicotemporales, refiriéndose a estos como la distancia presente desde su lugar de residencia a la unidad, sumándose a ello, la poca disponibilidad del personal trabajador de la UTCA.

Waller et al. (2009) afirman que los abandonos tardíos podrían estar relacionados con el progreso del tratamiento, la dificultad del mismo, y/o la propia mejoría. Esto último avala nuestra hipótesis y coincide con nuestros resultados, ya que un alto porcentaje de los abandonos tardíos están motivados principalmente por una mejoría del trastorno. Además, Waler et al., (2009) proponen que la impulsividad podría estar relacionada también con los abandonos precoces, característica que se halla presente en la muestra estudiada. En nuestra muestra el $70 \%$ son pacientes con un diagnóstico de TCANE, en el que algo más de la mitad (55\%) presentan un perfil bulímico, de los cuales la mitad están diagnosticados de TCANE con perfil bulímico, con rasgos de personalidad impulsiva, siendo estos, un posible motivo de abandono del tratamiento por iniciativa propia y contrario al consejo profesional, sin embargo, nuestros datos no evidencian que exista relación entre el diagnóstico y el tipo de abandono.

El $11 \%$ de pacientes dejó de acudir por motivos personales, entre los cuales se encontraban mayoritariamente embarazos y enfermedades propias o de familiares. Algunos de ellos referían que su TCA se había detenido durante un periodo de tiempo (la mayor parte por embarazo) otros sin embargo, seguían enfermos pero su situación personal hacía que el acudir a terapia fuese algo secundario.
Otros pacientes relataban sentir vergüenza por acudir, tener problemas económicos, no tener fuerza de voluntad, no tener suficiente seriedad, desesperanza, comorbilidad con otros trastornos, etc.

El 7.4\% de los abandonos está relacionado con las características del tratamiento o la relación terapéutica. En la literatura pocos estudios hacen referencia a este factor, sin embargo, Clinton (1996) observó que las diferencias de las expectativas entre el paciente y el terapeuta aumentaba el riesgo de abandono. Por tanto, parece importante que los terapeutas y los pacientes discutan abiertamente sobre las expectativas del tratamiento. Incluso consideramos que podría ser beneficioso que en los centros de salud mental, se dispusiera de tratamientos con diferentes enfoques terapéuticos. En muchas ocasiones, las expectativas creadas por los pacientes sobre el tipo de tratamiento que van a recibir, no se corresponden con las características que en la realidad se ofrecen.

Existe evidencia en el área de la investigación que muestra la existencia de diferentes motivos de abandono en los tratamientos en pacientes con TCA, entre ellos y los más estudiados, están la relación terapeuta-paciente (Clinton, 1996; Watson et al., 2013), sin embargo, la escasa literatura impide estudiar el tema en profundidad sobre otros motivos de abandono desde la percepción del paciente con TCA. Waller et al. (2009) refieren que el abandono del tratamiento tiene relación con el sexo: ser hombre y mujer; sin embargo parece que el hecho de ser varón o mujer y tener una edad u otra, no guarda relación con que un tratamiento psicológico sea más o menos prolongado (Ballesteros, Fernández, \& Labrador, 2013). Ante estos resultados y en pro de futuras investigaciones, sería relevante poder contar con un mayor número de sujetos clínicos, con el fin de controlar sesgos y la variabilidad debida a las diferencias individuales en cuanto a sexo y edad. Aunque consideramos que disponer de una muestra amplia de hombres resulta complicado, sería igualmente interesante, poder investigar la población masculina y descubrir las diferencias entre hombres y mujeres. 
Este estudio no está exento de limitaciones. En nuestra muestra no ha sido posible obtener resultados significativos en relación con el sexo debido al menor número de varones que han participado en nuestra investigación ni tampoco valorar si la duración del tratamiento puede ser motivo de abandono en pacientes con TCA. Sería deseable poder contar con una muestra más representativa con el fin de evitar sesgos. Por otro lado, al tratarse de una investigación cualitativa que empleó una entrevista no presencial, hecha por medio de una llamada telefónica, los datos recogidos pueden verse afectados, ya que probablemente se hayan producido pérdidas de información. Hay que tener presente que los pacientes se encontraban en diferentes lugares y/o circunstancias y en ocasiones en compañía de otras personas, lo que condicionaba la información obtenida y por lo tanto, la sesgaba. Adicionalmente, el instrumento utilizado para la recolección de datos carecía de fiabilidad y validez.

Por último, puesto que no existe gran literatura al respecto y el tópico es de gran relevancia, sería conveniente realizar un instrumento que recoja los diferentes motivos de abandono en los tratamientos de pacientes diagnosticados de TCA.

\section{Referencias}

American Psychiatric Association (2002). DSMIV-R. Manual diagnóstico y estadístico de los trastornos mentales. Barcelona: Masson.

Ballesteros, F., Fernández, P., \& Labrador, F. (2013). Factores que influyen en la duración de los tratamientos psicológicos empíricamente apoyados. Anales de psicología, 29, 94-102.

Beumont, P., Russell, J., \& Touyz, S. (1993). Treatment of anorexia nervosa. The Lancet , 341 , 1635-1640. http://dx.doi.org/10.101 6/0140-6736(93)90769-D

Clinton, D. (1996). Why Do Eating Disorder Patients Drop Out? Psychotherapy and
Psychosomatics, 65 , 29-35. http://dx.doi.o rg/10.1159/000289028

Currin, L., Schmidt, U., Treasure, J., \& Jick, H. (2005). Time trends in eating disorder incidence. The British Journal of Psychiatry, 186 (2), 132-135.

Gómez del Barrio, J.A., Gaite, L., Carral, L., Herrero C., \& Vázquez, J. (2012). Guía de Prevención de los Trastornos de la Conducta Alimentaria y el Sobrepeso - Dirección General de Ordenación y Atención Sanitaria. Gobierno de Cantabria.

Hoek, H. (2006). Indidence, prevalence and mortality of anorexia nervosa and other eating disorders. Current Opinion in Psychiatry, 19, 389-94.

Hoek, H., \& Van Hoeken, D. (2003). Review of the prevalence and incidence of eating disorders. International Journal of Eating Disorders, 34 , 383-96. http://dx.doi.org/1 0.1002/eat.10222

Hudson, J., Hiripi, E., Pope, H. \& Kessler, R. (2007). The prevalence and correlates of eating disorders in the national comorbidity survey replication. Biological Psychiatry, 61, 348-58. http://dx.doi.org/10.1016/j.bio psych.2006.03.040

Jones, D., Fox, M., Babigan, H., \& Hutton, H. (1980). Epidemiology of anorexia nervosa in Monroe County, New York, 1960-1976. Psychosomatic Medicine, 42, 551-558. http://dx.doi.org/10.1097/000068 42-198011000-00003

Kendell, R., Hall, D., Halley, A. \& Babigan, H. (1973). The epidemiology of anorexia nervosa. Psychological Medicine, 3 ,200-203. http://dx.doi.org/10.1017/S003 3291700048522

López, C. \& Treasure, J., (2011). Trastornos de la Conducta Alimentaria en adolescentes: Descripción y manejo. Revista Médica Clínica Condes, 22 , 85-97.

Pike, K., (1998). Long-term course of anorexia nervosa: Response, relapse, remission, and recovery. Clinical Psychology Review, 18 , 447-475.

Ryan, R., \& Deci, E. (2000). Self-determination theoryand the facilitation of intrinsic 
motivation, social development and wellbeing. American Psychologist, 55 , 68-78.

IBM. (2010). Statistical Package for the Social Sciences (Version 19.0). [Statistical Software]. Madrid: IBM.

Vandereychken, W., Soenens, B., \& Vansteenkiste M. (2009). Motivation to change in eating disorder patients: Aconceptual clari\#cation based on self-determination theory. International Journal of Eating Disorders, 37 ,207-219.

Velázquez, A., Sosa, J., Carmen, M., \& Sentíes, H. (2013). El abandono del tratamiento psiquiátrico: Motivos y contexto institucional. Revista de Psicología, 18, 315-340.

Vellisca, M.Y., Orejudo, S. \& Latorre, J.I. (2012). Distorsión de la Percepción Corporal en Pacientes con Anorexia Nerviosa de Inicio Temprano vs. Tardío. Clínica y Salud, 23 , 111-121.

Waller, G., Schmidt, U., Treasure, J., Murray, K., Alenya, J., \& Emanuelli, F. (2009) Problems across care pathways in specialist adult eating disorder services. Psychiatric Bulletin, 33 , 26-29. http://dx.doi.org/10.1 192/pb.bp.107.018325

Watson, H. J., Fursland, A., \& Byrne, S. (2013). Treatment Engagement in Eating Disorders: Who Exists Before Treatment? International Journal of Eating Disorder, $1,1-6$.

Zuroff, D., Koestner, R., \& Moskowitz, D. (2007). Autonomous motivation for therapy: A new non-specific predictor of outcome in brief treatments of depression. Psychotherapy Research, 17, 137-148.

\section{Notas}

* Artículo de investigación 